\title{
Galerkin - Kantorovich Method for the Elastic Buckling Analysis of Thin Rectangular SCSC Plates
}

\author{
Michael Ebie Onyia ${ }^{1}$, Eghosa. O. Rowland-Lato ${ }^{2}$ and Charles Chinwuba Ike ${ }^{3}$ \\ ${ }^{1}$ Department of Civil Engineering University of Nigeria, Nsukka, Enugu State, Nigeria. \\ ORCID: 0000-0002-0956-0077 \\ ${ }^{2}$ Department of Civil Engineering, University of Port Harcourt, Choba. Nigeria.
}

${ }^{3}$ Department of Civil Engineering, Enugu State University of Science and Technology, Enugu.

ORCID: 0000-0001-6952-0993

\begin{abstract}
This paper presents the elastic buckling analysis of thin rectangular plates using the Galerkin-Kantorovich method. The plate has two opposite simply supported edges $x=0$ and $x$ $=a$, and two clamped edges $y=0$ and $y=b$ where the origin is a corner and the uniform compressive load is applied at the simply supported edges. Mathematically, the problem is a boundary value problem (BVP) represented by a domain differential equation and boundary conditions. The buckling deflection function is assumed as an infinite series of an unknown function of $y$ coordinate $(G(y))$ and a known function (of the $x$ coordinate) that satisfies all the Dirichlet boundary conditions along the simply supported edges. The Galerkin-Kantorovich formulation of the BVP is an integral equation that further simplified to a homogeneous fourth order ordinary differential equation (ODE) in the unknown function $G(y)$. The general solution of the ODE was achieved using trial function methods. The enforcement of the boundary conditions along the clamped edges resulted in a system of homogeneous equations in terms of the four integration constants. The condition for nontrivial solution is used to obtain the characteristic buckling equation as a transcendental equation which is solved for the elastic buckling load for each buckling mode using computer software based iteration methods. The critical elastic buckling load is found to correspond to the first buckling mode. The characteristic elastic buckling equation obtained is found to be the exact buckling equation for the problem and is identical with previously obtained equations. The elastic buckling loads obtained agree with the previous results from the literature.
\end{abstract}

Keyword: Galerkin-Kantorovich method, elastic buckling load, characteristic elastic buckling equation, critical buckling equation, critical elastic buckling load, Kirchhoff plate.

\section{INTRODUCTION}

Plates are structural components extensively used to model aircraft wings, spacecraft panels, ship hulls and decks, building floors, bridge decks, roof slabs and offshore platforms. Though most plates perform satisfactorily under tensile forces, when they are subjected to in-plane compressive forces which may be uniform or non-uniform, they are prone to elastic buckling failures [1 - 13]. Usually, buckling of plates due to in-plane compressive forces which may be uniform or non-uniform is a nonlinear phenomenon which may occur suddenly leading to catastrophic structural failures. It is thus important to determine the load buckling capacities of plates as part of their design and analysis in order to avert premature failures.

Plate buckling may be classified as elastic buckling or plastic (inelastic) buckling. The buckling is classified as elastic buckling when the critical buckling stress is less than the elastic limit of the plate material. In practical problems the plate may be stressed beyond the elastic limit of the material before the commencement of buckling, and the buckling problem is called plastic (inelastic) buckling problem.

Navier derived the partial differential equation governing the elastic buckling problems of thin rectangular, isotropic, homogeneous plates under distributed transverse load and included the twisting term in the equation. Navier's work was particularly significant at the time because it was found that the twisting term can remarkably reduce the transverse deflections under distributed transverse loads. Saint Venant later modified the Navier's equation by including edge forces and shearing forces applied in the axial directions of the plate.

The differential equations derived independently by Navier and Saint Venant provided the foundations for a significant part of the research work on the elastic buckling of thin rectangular plates subjected to uniaxial, biaxial and (uniform and/or non-uniform) compressive loads and shear forces applied at the edges.

The basic problem of elastic buckling of plates is thus to find the minimum compressive forces/loads (buckling loads) and the associated shapes (buckling shapes) at which the plate would undergo unrestrained, excessive deformations when subjected to compressive loads (uniform or non-uniform) applied at the edges for known edge support conditions. The compressive loads may be applied uniaxially or biaxially or the loads may be shear forces $[4,10,11,12,13,14,15,16]$. Elastic buckling problems can be solved in closed form or by approximate numerical methods. 
Timoshenko [1, 2] solved the governing domain differential equation for the elastic buckling of thin rectangular isotropic homogeneous plates subjected to uniaxial uniform compressive load for different types of boundary conditions. He used the Navier's double trigonometric series method and the total potential energy minimization method to obtain the characteristic buckling equations, which he solved to find the buckling loads. He thus obtained elastic buckling loads and buckling modal shapes.

Kim et al [17] presented buckling analysis of isotropic and orthotropic plates using the two variable refined plate theory (RPT) which takes account of transverse shear deformation effects. The refined plate theory used by Kim et al [17] assumed a parabolic distribution of transverse shear strains through the plate thickness, thus obviating the necessity for the use of shear stress modification factors. They derived the governing equations using the principle of virtual displacements, and obtained closed-form solutions to the governing equations for a simply supported rectangular plate subjected to in-plane loads by the use of Navier's double trigonometric series method. They compared their numerical results with classical thin plate theory solutions, first order shear deformable plate solutions, and previously obtained exact solutions; and found their solutions to be comparable to the first order shear deformable plate theory.

Ibearugbulem [18] used the Ritz direct variational method to obtain the elastic buckling loads of thin rectangular flat plates subjected to uniaxial uniform compressive forces. He considered various boundary conditions for the plate (simply supported edges, clamped edges and plates with some edges clamped and the other edges simply supported). He derived one parameter shape functions for the various edge support conditions using the polynomial solution of deflections of Euler - Bernoulli beams with equivalent support conditions in the $x$ Cartesian coordinate directions. By substitution of the one-parameter displacement shape function obtained into the total potential energy functional for the elastic stability problem, and minimization with respect to unknown displacement parameter, he obtained solutions that were comparable to other previously obtained solutions for the problem.

Nwadike [19] also presented the Ritz method for the determination of the elastic buckling loads of isotropic, homogeneous, thin rectangular plates subjected to uniform compressive load in one coordinate direction. He considered various boundary conditions. He used the deflection functions for beams with equivalent end supports in the $x$ and $y$ Cartesian coordinates to generate one-parameter displacement functions for the plates for each considered boundary conditions. By substitution of the obtained one-parameter displacement functions into the total potential energy functional for the elastic stability problem, and minimization with respect to the unknown displacement parameter, he obtained solutions for the elastic buckling load for the boundary conditions considered. His solutions were comparable with previously obtained solutions, and the errors were not too big considering the simplification offered by the one-parameter displacement function used.

Oguaghamba [20] analysed the buckling and post-buckling loads of thin rectangular plates made of isotropic homogeneous materials. Oguaghamba et al [21] studied and presented the buckling and post-buckling loads characteristics of thin rectangular clamped plates made of isotropic, homogeneous materials.

Ibearugbulem et al [22] and Osadebe et al [23] used the truncated Taylor - Maclaurin's series to obtain displacement shape functions which were used in Galerkin variational method to determine the elastic buckling loads of simply supported thin rectangular plates subjected to uniform inplane compressive loads applied in one direction. They obtained solutions comparable with previous results in the literature.

Abodi [24] presented a finite difference method (FDM) for finding the elastic buckling loads of Kirchhoff plates subjected to in-plane patch loads. Shi [25] and Shi and Beziné [26] solved the elastic stability problem of orthogonally anisotropic thin rectangular plates using the boundary element method.

Nwoji et al [14] determined the elastic buckling loads of simply supported thin isotropic rectangular plates subjected to uniform compressive loads applied at the two opposite edges. They used the two dimensional finite Fourier sine integral transform method in solving the domain partial differential equation (PDE] for the elastic buckling problem. They found that the two dimensional integral transformation converts the PDE to an integral equation, and ultimately to an algebraic problem that gives exact solutions for the elastic buckling loads for all the buckling modes and also gives exact buckling modal shape functions.

Onah et al [15] used the one-dimensional finite Fourier sine integral transform method to determine the elastic buckling loads of Kirchhoff plate with two opposite edges clamped and the other two opposite edges simply supported with the two simply supported edges subjected to the uniform compressive force. They found that the one-dimensional integral sine transform converts the PDE representing the domain governing equation to an integral equation; which further was reduced to an ordinary differential equation. They obtained the general solution to the ODE and applied the boundary conditions along the clamped edges to express the problem as a system of homogeneous equations in terms of the four unknown integration constants. The condition for nontrivial solution of the homogeneous equations was used to obtain the characteristic buckling equations, which is a transcendental equation. They obtained the eigenvalues of the problem as the roots of the transcendental equation. Consequently, they obtained the exact solutions to the elastic buckling loads.

Several other seminal research papers on the theme of elastic and inelastic buckling of plates of various shapes, subjected to different boundary and loading conditions are reported in the literature. Some of these research works are reported by Batford and Houbolt [27], Wang et al [28], Ullah et al [29], Xiang et al [30], Ullah et al [31], Abolghasemi et al [32], Ezeh et al [33] and Ibearugbulem and Eze [34]. 
In this work, the Galerkin - Kantorovich method is used to obtain solutions for the elastic buckling loads of thin rectangular plates subjected to uniaxial uniform compressive force along the simply supported edges $x=0$ and $x=a$ and clamped along the edges $y=0$ and $y=b$.

\section{THEORETICAL FRAMEWORK}

The elastic buckling problem of rectangular Kirchhoff plates with in-plane dimensions of length $a$ and width $b$ is expressed by the partial differential equation (PDE):

$$
\begin{gathered}
D\left(\frac{\partial^{4} w(x, y)}{\partial x^{4}}+2 \frac{\partial^{4} w(x, y)}{\partial x^{2} \partial y^{2}}+\frac{\partial^{4} w(x, y)}{\partial y^{4}}\right)+N_{x} \frac{\partial^{2} w(x, y)}{\partial x^{2}}+N_{y} \frac{\partial^{2} w(x, y)}{\partial y^{2}}+ \\
2 N_{x y} \frac{\partial^{2} w}{\partial x \partial y}=0
\end{gathered}
$$

where $0 \leq x \leq a, \quad 0 \leq y \leq b, \quad x$ and $y$ are the in-plane Cartesian coordinates

$w(x, y)$ is the deflection

$N_{x}$ is the uniform compressive force in the $x$ direction

$N_{y}$ is the uniform compressive force in the $y$ direction

$N_{x y}$ is the twist

$D$ is the flexural rigidity of the plate material expressed by:

$$
D=\frac{E h^{3}}{12\left(1-\mu^{2}\right)}
$$

$E$ is the Young's modulus of elasticity of the plate material, $\mu$ is the Poisson's ratio of the plate material, $h$ is the plate thickness.

For the thin rectangular plate considered in this work, with simply supported edges $x=0$ and $x=a$, and edges $y=0$ and $y$ $=b$ clamped, the boundary conditions are:

$$
\begin{aligned}
& w(x=0, y)=w(x=a, y)=0 \\
& M_{x x}(x=0, y)=M_{x x}(x=a, y)=0 \\
& w(x, y=0)=w(x, y=b)=0 \\
& \frac{\partial w}{\partial y}(x, y=0)=\frac{\partial w}{\partial y}(x, y=b)=0
\end{aligned}
$$

\section{METHODOLOGY}

By the Galerkin - Kantorovich methodology, the deflection function is chosen in the variable separable form:

$$
w(x, y)=\sum_{n=1}^{\infty} G(y) \sin \frac{n \pi x}{a}
$$

where $G(y)$ is an unknown function of $y$ that is sought such that Equation (7) satisfies the domain PDE and $G(y)$ satisfies the boundary conditions along the edges $y=0$ and $y=b$.
In Equation (7) the sinusoidal function satisfies all the boundary conditions along the edges $x=0$, and $x=a$. The elastic buckling problem considered is shown in Figure 1,

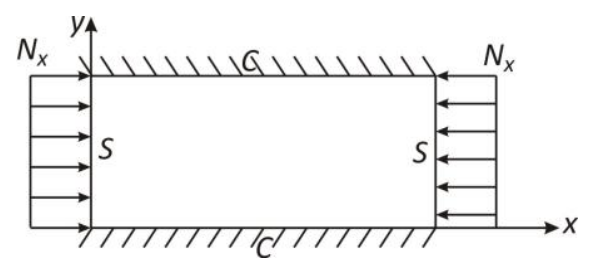

Figure 1: Elastic buckling of thin rectangular SCSC plate under uniaxial uniform compressive force $N_{x}$ along the simply supported edges $x=0$ and $x=a$

The governing PDE for the domain is a simplification of Equation (1) since $N_{y}=N_{x y}=0$, and we have:

$$
\begin{aligned}
& \nabla^{4} w+\frac{N_{x}}{D} \frac{\partial^{2} w}{\partial x^{2}}=0 \\
& \frac{\partial^{4} w}{\partial x^{4}}+2 \frac{\partial^{4} w}{\partial x^{2} \partial y^{2}}+\frac{\partial^{4} w}{\partial y^{4}}+\frac{N_{x}}{D} \frac{\partial^{2} w}{\partial x^{2}}=0 \\
& \text { where } \nabla^{4}=\frac{\partial^{4}}{\partial x^{4}}+2 \frac{\partial^{4}}{\partial x^{2} \partial y^{2}}+\frac{\partial^{4}}{\partial y^{4}}
\end{aligned}
$$

The Galerkin - Kantorovich variational integral statement of the problem is:

$$
\int_{0}^{a} \int_{0}^{b}\left\{\nabla^{4} \sum_{n=1}^{\infty} G(y) \sin \frac{n \pi x}{a}+\frac{N_{x}}{D} \frac{\partial^{2}}{\partial x^{2}} \sum_{n=1}^{\infty} G(y) \sin \frac{n \pi x}{a}\right\} \sin \frac{m \pi x}{a} d x d y=0
$$

Hence,

$$
\sum_{n=1}^{\infty} \int_{0}^{a} \int_{0}^{b}\left\{\nabla^{4} G(y) \sin \frac{n \pi x}{a}+\frac{N_{x}}{D} \frac{\partial^{2}}{\partial x^{2}} G(y) \sin \frac{n \pi x}{a}\right\} \sin \frac{m \pi x}{a} d x d y=0
$$

Simplifying,

$$
\begin{gathered}
\sum_{n=1}^{\infty} \int_{0}^{a} \int_{0}^{b}\left\{\left(\frac{n \pi}{a}\right)^{4} G(y) \sin \frac{n \pi x}{a}-2\left(\frac{n \pi}{a}\right)^{2} \frac{d^{2} G(y)}{d y^{2}} \sin \frac{n \pi x}{a}+\frac{d^{4}}{d y^{4}} G(y) \sin \frac{n \pi x}{a}-\right. \\
\left.\frac{N_{x}}{D}\left(\frac{n \pi}{a}\right)^{2} G(y) \sin \frac{n \pi x}{a}\right\} \sin \frac{m \pi x}{a} d x d y=0
\end{gathered}
$$

Further simplification gives:

$$
\begin{aligned}
& \sum_{n=1}^{\infty} \int_{0}^{b}\left\{\frac{d^{4} G(y)}{d y^{4}}-2\left(\frac{n \pi}{a}\right)^{2} \frac{d^{2} G(y)}{d y^{2}}+\left(\left(\frac{n \pi}{a}\right)^{4}-\frac{N_{x}}{D}\left(\frac{n \pi}{a}\right)^{2}\right) G(y)\right\} d y \times \\
& \int_{0}^{a} \sin \frac{n \pi x}{a} \sin \frac{m \pi x}{a} d x=0 \\
& \text { Let } \quad I_{m n}=\int_{0}^{a} \sin \frac{n \pi x}{a} \sin \frac{m \pi x}{a} d x
\end{aligned}
$$


Due to the orthogonality properties of the sine functions,

$I_{m n}=0$ if $m \neq n ; I_{m n}=a / 2$ if $m=n$

For non zero values of $I_{m n}, m=n$, and the problem simplifies to:

$\sum_{n=1}^{\infty} \int_{0}^{b}\left(\frac{d^{4} G(y)}{d y^{4}}-2\left(\frac{n \pi}{a}\right)^{2} \frac{\partial^{2} G(y)}{\partial y^{2}}+\left(\left(\frac{n \pi}{a}\right)^{4}-\frac{N_{x}}{D}\left(\frac{n \pi}{a}\right)^{2}\right) G(y)\right) d y=0$

Since $I_{m n} \neq 0$.

Equation (16) - holds only if:

$\frac{d^{4} G(y)}{d y^{4}}-2\left(\frac{n \pi}{a}\right)^{2} \frac{d^{2} G(y)}{d y^{2}}+\left(\left(\frac{n \pi}{a}\right)^{4}-\frac{N_{x}}{D}\left(\frac{n \pi}{a}\right)^{2}\right) G(y)=0$

Equation (17) is a fourth order homogeneous ordinary differential equation (ODE) in $G(y)$. The solution to Equation (17) is sought for in the exponential form.

Let $G(y)=\exp s y$

where $s$ is an undetermined parameter sought.

Then, the ODE becomes:

$\left\{s^{4}-2\left(\frac{n \pi}{a}\right)^{2} s^{2}+\left(\left(\frac{n \pi}{a}\right)^{4}-\frac{N_{x}}{D}\left(\frac{n \pi}{a}\right)^{2}\right)\right\} \exp s y=0$

For nontrivial solutions

$\exp s y \neq 0$, and the fourth degree polynomial results:

$s^{4}-2\left(\frac{n \pi}{a}\right)^{2} s^{2}+\left(\frac{n \pi}{a}\right)^{4}-\frac{N_{x}}{D}\left(\frac{n \pi}{a}\right)^{2}=0$

The roots are found from:

$\left(s^{2}-\left(\frac{n \pi}{a}\right)^{2}\right)^{2}=\frac{N_{x}}{D}\left(\frac{n \pi}{a}\right)^{2}$

The four roots are:

$\beta_{1}=\left(\left(\left(\frac{n \pi}{a}\right)^{2} \frac{N_{x}}{D}\right)^{1 / 2}+\left(\frac{n \pi}{a}\right)^{2}\right)^{1 / 2}$

$\beta_{2}=\left(\sqrt{\left(\left(\frac{n \pi}{a}\right)^{2} \frac{N_{x}}{D}\right)}-\left(\frac{n \pi}{a}\right)^{2}\right)^{1 / 2}$

The general solution for $G(y)$ is found as:

$G(y)=c_{1} \cosh \beta_{1} y+c_{2} \sinh \beta_{1} y+c_{3} \cos \beta_{2} y+c_{4} \sin \beta_{2} y$

\section{RESULTS}

The four boundary conditions along the edges $y=0$, and $y=b$ are used in the expression for $G(y)$ to set up a system of homogeneous equations. Hence from Equation (3)

$G(y=0)=0$
$G(y=b)=0$

$\frac{\partial G}{\partial y}(y=0)=0$

$\frac{\partial G}{\partial y}(y=b)=0$

$\frac{\partial G(y)}{\partial y}=c_{1} \beta_{1} \sinh \beta_{1} y+c_{2} \beta_{1} \cosh \beta_{1} y-c_{3} \beta_{2} \sin \beta_{2} y+c_{4} \beta_{2} \cos \beta_{2} y$

Equation (25) gives

$c_{1}+c_{3}=0$

Equation (27) gives:

$c_{2} \beta_{1}+c_{4} \beta_{2}=0$

Hence, from Equation (30), we have:

$c_{3}=-c_{1}$

From Equation (31), we have:

$c_{4}=-\frac{c_{2} \beta_{1}}{\beta_{2}}$

Equation (26) gives:

$G(y=b)=c_{1} \cosh \beta_{1} b+c_{2} \sinh \beta_{1} b+c_{3} \cos \beta_{2} b+c_{4} \sin \beta_{2} b=0$

Equation (28) gives:

$\frac{\partial G}{\partial y}(y=b)=c_{1} \beta_{1} \sinh \beta_{1} b+c_{2} \beta_{1} \cosh \beta_{1} b-c_{3} \beta_{2} \sin \beta_{2} b+c_{4} \beta_{2} \cos \beta_{2} b=0$

Using Equations (32) and (33) and rearranging of Equation (34) gives:

$c_{1}\left(\cosh \beta_{1} b-\cos \beta_{2} b\right)+c_{2}\left(\sinh \beta_{1} b-\frac{\beta_{1} \sin \beta_{2} b}{\beta_{2}}\right)=0$

Similarly using Equations (32) and (33) and rearranging Equation (35) gives:

$c_{1}\left(\beta_{1} \sinh \beta_{1} b+\beta_{2} \sin \beta_{2} b\right)+c_{2}\left(\beta_{1} \cosh \beta_{1} b-\beta_{1} \cos \beta_{2} b\right)=0$

In matrix form, we have

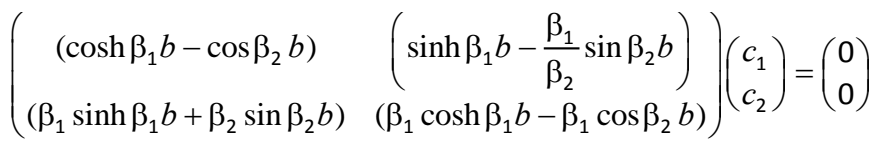

For nontrivial solutions,

$\left(\begin{array}{l}c_{1} \\ c_{2}\end{array}\right) \neq 0$

The characteristic elastic buckling equation is then: 
$\left|\begin{array}{cc}\left(\cosh \beta_{1} b-\cos \beta_{2} b\right) & \left(\sinh \beta_{1} b-\frac{\beta_{1}}{\beta_{2}} \sin \beta_{2} b\right) \\ \left(\beta_{1} \sinh \beta_{1} b+\beta_{2} \sin \beta_{2} b\right) & \beta_{1}\left(\cosh \beta_{1} b-\cos \beta_{2} b\right)\end{array}\right|=0$

Expansion of the determinant gives:

$\beta_{1}\left(\cosh \beta_{1} b-\cos \beta_{2} b\right)^{2}-\left(\sinh \beta_{1} b-\frac{\beta_{1}}{\beta_{2}} \sin \beta_{2} b\right)\left(\beta_{1} \sinh \beta_{2} b+\beta_{2} \sin \beta_{2} b\right)=0$

Further simplification gives the transcendental equation:

$2\left(1-\cosh \beta_{1} b \cos \beta_{2} b\right)+\left(\frac{\beta_{1}}{\beta_{2}}-\frac{\beta_{2}}{\beta_{1}}\right) \sinh \beta_{1} b \sin \beta_{2} b=0$

The elastic buckling loads are found by solving the transcendental equation using the computer based iteration methods. The solution to the transcendental equation for values of the plate aspect ratio $(a / b)$ ranging from 0.4 to 2.0 and for Poisson's ratio $\mu=0.25$ are calculated and presented in Table 1 in terms of the elastic buckling load coefficient $k(a / b)$ defined in terms of the dimensionless critical buckling load $\bar{N}_{c r}$ defined as:

$$
\bar{N}_{c r}=k\left(\frac{a}{b}\right)=\frac{N_{x} b^{2}}{D \pi^{2}}
$$

The critical buckling stress $\left(\sigma_{x x}\right)_{c r}$ is found as:

$$
\left(\sigma_{x x}\right)_{c r}=\frac{k(a / b) E}{12\left(1-\mu^{2}\right)}\left(\frac{h}{b}\right)^{2}
$$

Table 1: Critical elastic buckling load coefficients $k(a / b)\left(=\bar{N}_{c r}\right)$ of thin rectangular plates clamped along the edges $y=0$, and $y=b$ and subjected to uniform compressive load $N_{x}$ along the simply supported edges $x=0$, and $x=a$

\begin{tabular}{|c|c|c|c|c|c|c|c|c|c|}
\hline$a / b$ & 0.4 & 0.6 & 0.8 & 1.0 & 1.2 & 1.4 & 1.6 & 1.8 & 2.0 \\
\hline $\begin{array}{c}k(a / b)=\bar{N}_{c r} \\
\left(=\frac{N_{x x} b^{2}}{D \pi^{2}}\right)\end{array}$ & 9.448 & 7.055 & 7.304 & 7.691 & 7.055 & 7.001 & 7.304 & 7.055 & 6.972 \\
\hline $\begin{array}{c}k(a / b) \text { exact Ohah et al [15] } \\
\text { Wang et al [10] }\end{array}$ & 9.448 & 7.055 & 7.304 & 7.691 & 7.055 & 7.001 & 7.304 & 7.055 & 6.972 \\
\hline Bulson [7] & 9.448 & 7.055 & 7.304 & 7.691 & 7.055 & 7.001 & 7.304 & 7.055 & 6.972 \\
\hline $\begin{array}{c}\text { Timoshenko and Gere [6] } \\
\text { Timoshenko [1, 2] }\end{array}$ & 9.448 & 7.055 & 7.304 & 7.691 & 7.055 & 7.001 & 7.304 & 7.055 & 6.972 \\
\hline
\end{tabular}

\section{DISCUSSION}

The Galerkin - Kantorovich method has been successfully used in this paper to solve the elastic buckling problem of a thin rectangular plate under uniform compressive force $N_{x}$ applied at the simply supported edges $x=0$, and $x=a$, while the other two edges $y=0$, and $y=b$ are clamped. The elastic stability problem is mathematically represented by the fourth order ordinary differential equation (ODE) - Equation (9) valid on the domain $(0 \leq x \leq a ; 0 \leq y \leq b)$ and the boundary conditions given by Equations (3) - (6).

Following the Galerkin - Kantorovich methodology, the deflection function is represented in the variable - separable form expressed by the infinite single series in Equation (7). The chosen deflection function Equation (7) satisfies apriori all the Dirichlet boundary conditions along the simply supported edges $x=0$, and $x=a$; but contains an unknown function of the $y$ Cartesian coordinate variable which is sought such that the domain equation is satisfied at all parts on the domain.

In the Galerkin - Kantorovich methodology, the domain ODE is expressed as an integral equation. The Galerkin Kantorovich variational integral statement of the elastic stability problem is expressed as the integral equation in Equation (11), which upon simplification gave Equation (14).
Further simplification of the Galerkin - Kantorovich variational integral statement of the elastic buckling problem obtained by the orthogonality properties of the sinusoidal functions and the fact that $I_{m n} \neq 0$, resulted in Equation (16). For any buckling mode, $n$, the integral in Equation (16) vanishes only when the problem reduces to the ODE in Equation (17).

Equation (17) is a fourth order ODE in terms of $G(y)$, which is solved using methods of variation of parameters, differential operators or trial functions to obtain the general solution for $G(y)$ as the equation set out in Equation (24) which has four constants of integration. The four constants of integration are determined upon enforcement of the four boundary conditions along the clamped edges $y=0$ and $y=b$. The application of the four boundary conditions yielded a system of four homogeneous equations - Equations (30), (31), (34) and (35) in terms of the four integration constants. Further simplification of the system of homogeneous equations using Equations (32) and (33) yielded the system of two homogeneous equations - Equations (36) and (37) which is expressed in matrix form as Equation (38). The characteristic elastic buckling equation is obtained from the condition for nontrivial solution as Equation (39) which is a determinantal equation. Expansion of the determinant of the characteristic stability equation gave Equation (40) which was further 
simplified to the transcendental equation - Equation (41). Equation (41) was solved using computer based iteration methods to obtain the elastic buckling loads for values of the Poisson's ratio $\mu=0.25$, and for various values of the plate aspect ratio $(a / b)$ ranging from 0.40 to 2.0. The results are presented in Table 1 together with results from the previous studies done by Wang et al [10], Bulson [7], Onah et al [15], Timoshenko [1, 2] and Timoshenko and Gere [6]. Table 1 reveals that the results obtained for critical elastic buckling loads using the Galerkin - Kantorovich method are identical with previous results obtained by Wang et al [10], Bulson [7], Timoshenko [1, 2], Timoshenko and Gere [6] and Onah et al [15].

\section{CONCLUSION}

In conclusion,

(i) The Galerkin - Kantorovich method presented in this paper is ideally suitable for the solution of the elastic stability problem of thin rectangular isotropic plate with clamped edges $(y=0$, and $y=b)$ and the simply supported edges $(x=0, x=a)$ subjected to uniform compressive force $N_{x}$.

(ii) The Galerkin - Kantorovich method assumes the buckling deflection function as an infinite series of the product of an unknown function $(G(y))$ and a known function of the $x$ coordinate which satisfies all the Dirichlet boundary conditions along the simply supported edges.

(iii) The Galerkin - Kantorovich variational integral is an integral equation and the BVP of elastic stability is thus converted to an integral equation over the domain of the plate $(0 \leq x \leq a, 0 \leq y \leq b)$.

(iv) The resulting integral equation is further simplified to a homogeneous fourth order ODE in the unknown function $G(y)$.

(v) The solution of the homogeneous fourth order ODE gives the general solution for $G(y)$ in terms of four unknown integration constants.

(vi) The application and enforcement of four boundary conditions to $G(y)$ along the clamped edges $y=0$, and $y$ $=b$ results to a system of four homogeneous equations in terms of the four integration constants. The system of four homogeneous equations is further simplified to a system of two homogeneous equations (by expressing two constants in terms of the other two constants).

(vii) The condition for nontrivial solution is used to obtain the characteristic elastic stability equation as a transcendental equation which is solved for $N_{x}$ using computational software tools and iteration methods.

(viii) The critical elastic buckling mode is found to correspond to the first buckling mode, when $n=1$.

(ix) The critical elastic buckling loads obtained using Galerkin - Kantorovich method were identical with the previous results obtained by Onah et al [15], Wang [10] and Timoshenko and Gere [6] who used other methods for solving the problem.

(x) The Galerkin - Kantorovich method gave exact solution for the critical elastic buckling load of SCSC or CSCS Kirchhoff plates under uniform compressive force $N_{x}$ applied at the simply supported edges $(x=0$, and $x=a)$

\section{REFERENCES}

[1] Timoshenko (1925). Applied Elasticity D Van Nostrand Co. Inc. New York.

[2] Timoshenko S. (1936). Theory of elastic stability. McGraw Hill Publishing Co Ltd https//doi.org/10.1007/S0368393100104389.

[3] Yoo C.H. and Lee S.C. (2011). Stability of Structures, Principles and Applications. Amsterdam Butterworth Heinemann.

[4] Iyengar N.G.R. (1988). Structural stability of columns and plates. Chichester England, Ellis Horward Halsted Press.

[5] Bouazza M., Quinas D., Yazid A. and Hamouine A. (2012). "Buckling of thin plates under uniaxial and biaxial compression" Journal of Materials Science and Engineering 32(8) (2012) pp 487 - 492.

[6] Timoshenko S.P. and Gere J.M. (1955). Theory of elastic stability. McGraw Hill, New York.

[7] Bulson P.S. (1970). The stability of flat plates. Chatto and Windus London, UK.

[8] Gambhir M.L. (2004). Elastic buckling of thin plates In: Stability Analysis and Design of Structures, Springer Berlin Heidelberg. https://doi.org/10.1007/978-3-66209996-4-8.

[9] Chajes A. (1993). Principles of structural stability theory. Englewood Cliffs New Jersey, Prentice Hall.

[10] Wang C.M., Wang C.Y., and Reddy J.N. (2005). Exact solutions for buckling of structural members. Boca Raton Florida CRC Press LLC.

[11] Yu Chen (2003). Buckling of rectangular plates under intermediate and end loads. MSc Thesis Department of Civil Engineering, National University of Singapore.

[12] Chattopadhyay A.P (2011). Elastic buckling behaviour of plate and tubular structures. MSc Thesis, Department of Mechanical and Nuclear Engineering, Kansas State University, Manhattan, Kansas.

[13] Ion Dima (2015). Buckling of flat plates under combined loading. Incas Bulletin Vol 7 Issue 1 pp $83-$ 96.

[14] Nwoji C.U., Onah H.N., Mama B.O., Ike C.C., Ikwueze E.U. (2017). Elastic buckling analysis of simply supported thin plates using the double finite Fourier sine integral transform method. Explorematics Journal of Innovative Engineering and Technology Vol 01 No 01 September 2017 pp $37-47$.

[15] Onah H.N., Nwoji C.U., Ike C.C. and Mama B.O. 
(2018). Elastic buckling analysis of uniaxilly compressed CCSS Kirchhoff plate using single finite Fourier sine integral transform method. Modelling Measurement and Control B Volume 87, No 2, June 2018, pp 107 -111. DOI: 10.18280/mmc.b.870208.

[16] Helbig D., Da Silva C.C.C., Real M. de V., Dos Santos E.D., Isoldi L.A., Rocha L.A.O. (2016). Study about buckling phenomenon in perforated thin steel plates employing computational modeling and constructal design method. Latin American Journal of Solids and Structures Vol 13 No 10 October 2016 pp 1912 - 1936. https//doi.org/10.1590/1679-78252893.

[17] Kim S.E., Thai H.T. and Lee J. (2009). "Buckling analysis of plates using the two variable refined plate theory." Thin - Walled Structures Volume 47 Issue 4, $\begin{array}{llllll}\text { April } & 2009 & \text { pp } & 455 & - & 462 .\end{array}$ https://doi.org/10.1016/j.tws.2008.08.002.

[18] Ibearugbulem O.M. (2012). Application of a direct variational principle in elastic stability analysis of thin rectangular flat plates. $\mathrm{PhD}$ Thesis Structural Engineering School of Post Graduate Studies, Federal University of Technology Owerri, May 2012.

[19] Nwadike A.N. (2014). Buckling analysis of isotropic rectangular plates using Ritz method. M. Eng Project report, Department of Civil Engineering, Federal University of Technology Owerri, July 2014.

[20] Oguaghamba O.A. (2015). Analysis of buckling and post buckling loads of isotropic thin rectangular plates. PhD Thesis Structural Engineering School of Post Graduate Studies, Federal University of Technology Owerri Nigeria.

[21] Oguaghamba O.A., Ezeh J.C., Ibearugbulem M.O. and Ettu L.O. (2015). Buckling and post buckling loads characteristics of all edges clamped thin rectangular plate. The International Journal of Sciences (IJES) Vol 4 Issue 11 pp $55-61$.

[22] Ibearugbulem O.M., Osadebe N.N., Ezeh J.C., Onwuka D.O. (2011). "Buckling analysis of axially compressed SSSC thin rectangular plate using Taylor - Maclaurin shape function." International Journal of Civil and Structural Engineering Vol 2 Issue 2, pp 676-681.

[23] Osadebe N.N., Nwokike V.C. and Oguaghamba O.A. (2016). Stability analysis of SSSS thin rectangular plate using multi-degrees of freedom. Taylor - Maclaurins series in Galerkin variational method. Nigerian Journal of Technology (NIJOTECH) Vol 35 No 3 July 2016. pp 503 - 509. http://dx.doi.org/10.4314/nijt/v.35i3.5.

[24] Abodi J.T. (2012). Elastic buckling analysis of plates under in-plane patch loading using finite difference method. Journal of Kerbala University, Vol 10 No 2, Scientific 2012 pp $142-150$.

[25] Shi G. (1990). Flexural vibration and buckling analysis of orthotropic plates by the boundary element method. International Journal of Solids and Structures 26:1351 1370.

[26] Shi G. and Bezine G.P. (1990). Buckling analysis of orthotropic plates by boundary element method. Mechanics Research Communications 17: $1-8$.

[27] Batford S.B. and Houbolt J.C. (1946). Critical combination of shear and transverse direct stress for an infinitely long flat plate with edges elastically restrained against rotation. NACA Report 847, $8-12$..

[28] Wang C.M., Xiang Y., and Chakrabarty J. (2001). Elastic/plastic buckling of thick plates. International Journal of Solids and Structures Vol 38: 8617 - 8640.

[29] Ullah S., Zhang J., Zhong U. (2020). New analytic solutions of buckling problem of rotationally restrained rectangular thin plates. International Journal of Applied Mechanics. DOI:10.1142/S1758825119501011.

[30] Xiang Y., Wang C.M. and Wang C.Y. (2001). Buckling of rectangular plates with an internal hinge. International Journal of Structural Stability and Dynamics Vol 1 No 2, 169 - 179.

[31] Ullah S, Wang H., Zhang X., Zhang J., Zhong Y, Li R. (2019). New analytic buckling solutions of moderately thick clamped rectangular plates by a straight forward finite integral transform method. Archive of Applied Mechanics September 2019, Volume 89, Issue 9, pp 1885 - 1897.

[32] Abolghasemi S., Eipakchi H., and Shariati M. (2019). An analytical solution for buckling of plates with circular cut out subjected to non-uniform in-plane loading. Archive of Applied Mechanics (2019) 89 Issue 12, 2519 - 2543. https://doi.org/10.1007/S00419-01901592-3.

[33] Ezeh J.C., Ibearugbulem M. Owus, Opara H.E., Oguaghamba O.A. (2014). Galerkin's indirect variational method in elastic stability analysis of all edges clamped thin rectangular plates. International Journal of Research in Engineering (IJRET) Vol 03, Issue 04 pp $674-679$.

[34] Ibearugbulem O.M. and Ezeh J.C. (2013). Instability of axially compressed CCCC thin rectangular plate using Taylor - Maclaurin's series shape function on Ritz method. Academic Research International Vol 4 Issue 1 , pp $346-351$. 